\title{
CP Violation in Correlated Production and Decay of Unstable Particles
}

\author{
Olaf $\operatorname{Kittel}^{a, b, c, 1]}$ AND Apostolos Pilaftsis ${ }^{b, c, d, 2}$ \\ ${ }^{a}$ Departamento de Física Teórica y del Cosmos and CAFPE, Universidad de Granada, \\ E-18071 Granada, Spain \\ ${ }^{b}$ Consortium for Fundamental Physics, School of Physics and Astronomy, \\ University of Manchester, Manchester M13 9PL, United Kingdom \\ ${ }^{c}$ Theory Division, CERN, CH-1211 Geneva 23, Switzerland \\ ${ }^{d}$ Department of Theoretical Physics and IFIC, University of Valencia-CSIC, \\ E-46100, Valencia, Spain
}

\begin{abstract}
We study resonant CP-violating Einstein-Podolsky-Rosen correlations that may take place in the production and decay of unstable scalar particles at high-energy colliders. We show that as a consequence of unitarity and CPT invariance of the S-matrix, in $2 \rightarrow 2$ scatterings mediated by mixed scalar particles, at least three linearly independent decay matrices associated with the unstable scalar states are needed to obtain non-zero CP-odd observables that are also odd under C-conjugation. Instead, for the correlated production and decay of two unstable particle systems in $2 \rightarrow 4$ processes, we find that only two independent decay matrices are sufficient to induce a net non-vanishing CP-violating phenomenon. As an application of this theorem, we present numerical estimates of CP asymmetries for the correlated production and decay of supersymmetric scalar top-antitop pairs at the LHC, and demonstrate that these could reach values of order one. As a byproduct of our analysis, we develop a novel spinorial trace technique, which enables us to efficiently evaluate lengthy expressions of squared amplitudes describing the resonant scalar transitions.
\end{abstract}

KEYWORDS: EPR correlation; resonant CP violation; collider phenomenology.

\footnotetext{
${ }^{1}$ Email: kittel@th.physik.uni-bonn.de

${ }^{2}$ Email: apostolos.pilaftsis@manchester.ac.uk
} 


\section{Introduction}

In 1935, Einstein, Podolsky and Rosen (EPR) [1] proposed a remarkable experiment, by which the non-local nature of quantum mechanics could be tested in unstable systems decaying into two entangled states. Contrary to authors' initial expectations, subsequent EPR-type experiments based on polarization of photons have refuted local realism, vindicating the non-local and non-causal interpretation of the quantum-mechanical wavefunction. The EPR paradigm has found numerous applications in modern quantum theory [2], such as quantum information, quantum dots and particle physics, including tests of CPT violation and quantum decoherence in $\phi$-factories as predicted in certain theories of quantum gravity [3].

In this paper, we study the physical consequences of EPR entanglement on resonant $\mathrm{CP}$ violation [4] in the correlated production and decay of unstable particles at high-energy colliders, such as the TEVATRON and the CERN pp Large Hadron Collider (LHC) (for related considerations at future colliders, see [5]). As a prototype example, we consider the pair production of SU(3)-coloured scalar particles $\widetilde{S}_{1,2}$ via virtual gluons at the LHC, followed by their decay into fermions, e.g. $p p \rightarrow \widetilde{S}_{1,2} \widetilde{S}_{1,2}^{*} \rightarrow(f \tilde{f})\left(\bar{f}^{\prime} \overline{\tilde{f}}^{\prime}\right)$. For instance, in supersymmetric theories, such coloured scalar particles could be scalar top or bottom

quarks, i.e. $\widetilde{S}_{1,2} \in\left\{\tilde{t}_{1,2}, \tilde{b}_{1,2}\right\}$, which can decay into the gauginos or Higgsinos $\tilde{f}, \overline{\tilde{f}}^{\prime}$ and Standard Model (SM) fermions $f, \bar{f}^{\prime}$. As a consequence of cross-correlation between unitarity and CPT invariance, we find that the two decaying 'arms' of $\widetilde{S}_{1,2}$ and $\widetilde{S}_{1,2}^{*}$ are not independent of each other. As we will show, at least two linearly independent decay channels are needed to obtain non-zero CP asymmetries. The proof of this statement gets facilitated with the use of a new trace technique, which we introduce in order to efficiently evaluate lengthy scalar amplitude expressions.

The paper is organized as follows. After this brief introduction, in Section 2 we review the formalism for describing $2 \rightarrow 2$ resonant scatterings, mediated by a system of mixed, unstable scalar particles, and present the action of $\mathrm{CP}$ and $\mathrm{T}$ transformations on the squared amplitudes. In Section 3 we discuss the unitarity and CPT constraints on CP-violating observables. Section 4 shows the connection between $2 \rightarrow 2$ and $2 \rightarrow$ 4 scatterings, and presents numerical estimates of $\mathrm{CP}$ asymmetries that may occur in the correlated production and decay of supersymmetric scalar top-anti-top pairs. In particular, the scattering processes are identified for optimally testing $\mathrm{CP}$ violation in the scalar top sector of the Minimal Supersymmetric extension of the Standard Model (MSSM) at the LHC. Section 5 contains our conclusions. In Appendix A, we give technical details of a novel spinorial trace technique which we develop to analytically evaluate lengthy expressions of amplitudes squared describing transitions mediated by unstable scalar particles.

\section{Formalism for Scalar Particle Mixing}

In this section, we will first review and then extend the former formalism for unstable particle mixing, presented in [4]. Our aim is to study CP asymmetries in the production and decay of scalar particles. Motivated by supersymmetry, we consider as a prototype example coloured scalar particles $\widetilde{S}_{\alpha}$, such as scalar top $\left(\tilde{t}_{1,2}\right)$ or bottom $\left(\tilde{b}_{1,2}\right)$ quarks. We assume that these particles are sufficiently heavy, so as to decay into two fermions, 


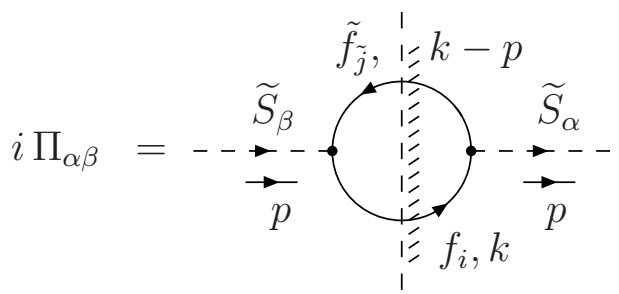

Figure 1: Scalar transitions $\widetilde{S}_{\beta} \rightarrow \widetilde{S}_{\alpha}$ at the one-loop level. A cut on the graph according to the Cutkosky rules [11] yields the absorptive contributions.

e.g. $\widetilde{S}_{\alpha} \rightarrow f_{i} \tilde{f}_{\tilde{j}}$, where $f_{i} \in\{t, b\}$ and $\tilde{f}_{\tilde{j}} \in\left\{\tilde{g}, \tilde{\chi}_{1,2,3,4}^{0}, \tilde{\chi}_{1,2}^{+}\right\}$. The generic Lagrangian describing the relevant scalar-fermion-gaugino/Higgsino interactions $\widetilde{S}_{\alpha^{-}} f_{i^{-}} \tilde{f}_{\tilde{j}}$ reads:

$$
\mathscr{L}_{\tilde{S}_{\alpha} f_{i} \tilde{f}_{\tilde{j}}}=\widetilde{S}_{\alpha} \bar{f}_{i}\left(C_{i \tilde{j}}^{\alpha, L} P_{L}+C_{i \tilde{j}}^{\alpha, R} P_{R}\right) \tilde{f}_{\tilde{j}}+\text { H.c. },
$$

with $P_{L, R}=\left(1 \mp \gamma_{5}\right) / 2$. Note that explicit couplings for $\widetilde{S}_{\alpha}=\tilde{t}_{\alpha}, \tilde{b}_{\alpha}$ in the CP-violating MSSM may be found, for example, in [9, 10].

The dynamics of the mixed $\widetilde{S}_{1} \widetilde{S}_{2}$ system may be described by the (unrenormalized) inverse scalar propagator matrix [4]

$$
\Delta_{0}^{-1}(s)=\left(\begin{array}{cc}
s-M_{1}^{2}+\Pi_{11}(s) & \Pi_{12}(s) \\
\Pi_{21}(s) & s-M_{2}^{2}+\Pi_{22}(s)
\end{array}\right) .
$$

The propagator matrix arises from summing up a geometric series of the $\widetilde{S}_{\alpha} \widetilde{S}_{\beta}$ self-energies $\Pi_{\alpha \beta}(s)$, as shown in Fig. 1, The self-energies may be linearly decomposed in their dispersive and absorptive parts as follows:

$$
\Pi_{\alpha \beta}(s)=\Pi_{\alpha \beta}^{\mathrm{disp}}(s)+i \Pi_{\alpha \beta}^{\mathrm{abs}}(s) .
$$

The dispersive part of the self-energy, $\Pi_{\alpha \beta}^{\text {disp }}(s)$, is UV infinite and requires renormalization. To this end, we consider the on-shell (OS) scheme, in which the dispersive parts satisfy the renormalization conditions [4]

$$
\Pi_{\alpha \beta}^{\mathrm{disp}}\left(M_{\alpha}^{2}\right)=\Pi_{\alpha \beta}^{\mathrm{disp}}\left(M_{\beta}^{2}\right)=0, \quad \lim _{s \rightarrow M_{\alpha}^{2}} \frac{\Pi_{\alpha \alpha}^{\mathrm{disp}}(s)}{s-M_{\alpha}^{2}}=0 .
$$

The advantage of the OS scheme is that around the resonant region $s \approx M_{1}^{2} \approx M_{2}^{2}$, the OSrenormalized, UV-finite dispersive self-energies are negligible, and only the absorptive selfenergies $\Pi_{\alpha \beta}^{\mathrm{abs}}(s)$ become relevant. Hence, one may obtain a Born-improved approximation for the propagator matrix given by

$$
\Delta^{-1}(s)=\left(\begin{array}{cc}
s-M_{1}^{2}+i \Pi_{11}^{\mathrm{abs}}(s) & i \Pi_{12}^{\mathrm{abs}}(s) \\
i \Pi_{21}^{\mathrm{abs}}(s) & s-M_{2}^{2}+i \Pi_{22}^{\mathrm{abs}}(s)
\end{array}\right) .
$$



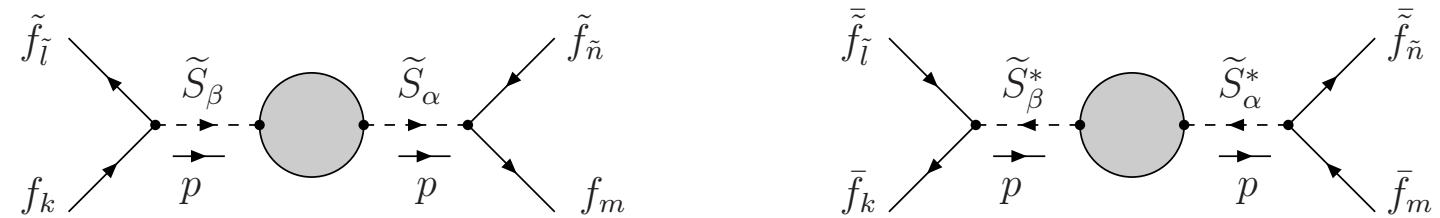

Figure 2: Transition amplitude $f_{k} \tilde{f}_{\tilde{l}} \rightarrow f_{m} \tilde{f}_{\tilde{n}}$ via the scalars $\widetilde{S}_{\alpha, \beta}$ (left), and its CPconjugate amplitude (right). The grey circles indicate one-loop resummed contributions.

Employing the standard Cutkosky cutting rules [11] as shown in Fig. 1, we can calculate the absorptive part of the self-energies, $\Pi_{\alpha \beta}^{\mathrm{abs}}(s)$. For our generic theory with scalar interactions $\widetilde{S}_{\alpha^{-}} f_{i^{-}}-\tilde{f}_{\tilde{j}}$, we obtain at the one-loop level,

$$
\begin{aligned}
& \Pi_{\alpha \beta}^{\mathrm{abs}}(s)=\frac{1}{16 \pi s} \lambda^{1 / 2}\left(s, m_{i}^{2}, m_{\tilde{j}}^{2}\right) \theta\left(\sqrt{s}-m_{i}-m_{\tilde{j}}\right) \\
& \quad \times\left[\left(C_{i \tilde{j}}^{\alpha, L *} C_{i \tilde{j}}^{\beta, L}+C_{i \tilde{j}}^{\alpha, R *} C_{i \tilde{j}}^{\beta, R}\right)\left(s-m_{i}^{2}-m_{\tilde{j}}^{2}\right)-2\left(C_{i \tilde{j}}^{\alpha, R *} C_{i \tilde{j}}^{\beta, L}+C_{i \tilde{j}}^{\alpha, L *} C_{i, \tilde{j}}^{\beta, R}\right) m_{i} m_{\tilde{j}}\right],
\end{aligned}
$$

where $\lambda(x, y, z)=(x-y-z)^{2}-4 y z$ and summation over all intermediate OS states $f_{i} \tilde{f}_{\tilde{j}}$ is implicitly assumed. Observe that the absorptive self-energies $\Pi_{\alpha \beta}^{\text {abs }}(s)$ are related to the anti-Hermitian part of the inverse propagator matrix (2.5), through

$$
\Pi_{\alpha \beta}^{\mathrm{abs}}(s)=-\frac{i}{2}\left[\Delta^{-1}(s)-\left(\Delta^{-1}(s)\right)^{\dagger}\right]_{\alpha \beta} .
$$

The latter is also related through the optical theorem to

$$
\left[\Delta^{-1}(s)-\left(\Delta^{-1}(s)\right)^{\dagger}\right]_{\alpha \beta}=i \sum_{X} \int \mathrm{dPS}_{X} V_{X}^{\alpha}\left(V_{X}^{\beta}\right)^{\dagger},
$$

where the sum is over all OS intermediate states $X$. In addition, $V_{X}^{\alpha, \beta}$ are the vertex amplitudes for the decay processes $\widetilde{S}^{\alpha, \beta} \rightarrow X$, and $\mathrm{PS}_{X}$ is the respective Lorentz-invariant phase space. Further constraints from unitarity and CPT invariance will be discussed in the next section.

It is now instructive to consider $2 \rightarrow 2$ scatterings, mediated by the coloured scalar particles $\widetilde{S}^{\alpha}$ in the $s$-channel, as shown in Fig. 2, Specifically, the resonant $s$-dependent part of the amplitude $A \rightarrow B$ is given by

$$
\mathcal{T}_{s}^{A \rightarrow B}(s)=\sum_{\alpha, \beta}\left(V_{B}^{\alpha}\right)^{*} \Delta_{\alpha \beta}(s) V_{A}^{\beta} \equiv V_{B}^{\dagger} \Delta(s) V_{A}
$$

where we use matrix notation in the space of the unstable scalar particles $\widetilde{S}^{\alpha}$ in the last step of the equation. Moreover, the vertex functions, for the in-state $A=f_{k} \tilde{f}_{\tilde{l}}$ and the 
out-state $B=f_{m} \tilde{f}_{\tilde{n}}$, have the analytic forms

$$
\begin{gathered}
V_{A}^{\beta} \equiv V_{k \tilde{l}}^{\beta}=\bar{v}\left(p_{\tilde{l}}, \lambda_{\tilde{l}}\right)\left(C_{k \tilde{l}}^{\beta, R *} P_{L}+C_{k \tilde{l}}^{\beta, L *} P_{R}\right) u\left(p_{k}, \lambda_{k}\right), \\
\left(V_{B}^{\alpha}\right)^{*} \equiv\left(V_{m \tilde{n}}^{\alpha}\right)^{*}=\bar{u}\left(p_{m}, \lambda_{m}\right)\left(C_{m \tilde{n}}^{\alpha, L} P_{L}+C_{m \tilde{n}}^{\alpha, R} P_{R}\right) v\left(p_{\tilde{n}}, \lambda_{\tilde{n}}\right),
\end{gathered}
$$

where $p_{k}$ and $\lambda_{k}$ symbolize the 4-momentum and the helicity for a given fermion $f_{k}$. It now proves useful to define the Hermitian matrix

$$
\begin{aligned}
T_{A}^{\beta \gamma}(s) \equiv \sum_{\lambda_{k}, \lambda_{\tilde{l}}} V_{A}^{\beta}\left(V_{A}^{\gamma}\right)^{*}= & \left(C_{k \tilde{l}}^{\beta, L *} C_{k \tilde{l}}^{\gamma, L}+C_{k \tilde{l}}^{\beta, R *} C_{k \tilde{l}}^{\gamma, R}\right)\left(s-m_{k}^{2}-m_{\tilde{l}}^{2}\right) \\
& -2\left(C_{k \tilde{l}}^{\beta, R *} C_{k \tilde{l}}^{\gamma, L}+C_{k \tilde{l}}^{\beta, L *} C_{k \tilde{l}}^{\gamma, R}\right) m_{k} m_{\tilde{l}},
\end{aligned}
$$

for the production vertex, including an analogous definition $T_{B}^{\beta \gamma}$ for the decay vertex. In terms of these matrices, the squared amplitude for the $s$-channel resonant process $A \rightarrow \widetilde{S}_{\alpha} \rightarrow B$ takes on the simple form:

$$
\left|\mathcal{T}_{s}^{A \rightarrow B}(s)\right|^{2}=T_{B}^{\delta \alpha}(s) \Delta_{\alpha \beta}(s) T_{A}^{\beta \gamma}(s) \Delta_{\gamma \delta}^{\dagger}(s)=\operatorname{Tr}\left[T_{B}(s) \Delta(s) T_{A}(s) \Delta^{\dagger}(s)\right] .
$$

Notice that the squared amplitude can be compactly written in the form of a trace over the scalar-particle space $\widetilde{S}_{\alpha}$.

For the CP-conjugate process $\bar{A} \rightarrow \widetilde{S}_{\alpha}^{*} \rightarrow \bar{B}$, the transition amplitude may be written down as follows:

$$
\mathcal{T}_{s}^{\bar{A} \rightarrow \bar{B}}=\left(\bar{V}_{B}^{\alpha}\right)^{*} \Delta_{\alpha \beta}^{\top}(s) \bar{V}_{A}^{\beta}=\bar{V}_{B}^{\dagger} \Delta^{\top}(s) \bar{V}_{A},
$$

where the $\mathrm{CP}$-conjugate in- and out-state vertex functions are given by

$$
\begin{aligned}
\left(\bar{V}_{B}^{\alpha}\right)^{*} & =\bar{v}\left(\bar{p}_{\tilde{n}}, \lambda_{\tilde{n}}\right)\left(C_{m \tilde{n}}^{\alpha, R} P_{L}+C_{m \tilde{n}}^{\alpha, L *} P_{R}\right) u\left(\bar{p}_{m}, \lambda_{m}\right)=V_{B}^{\alpha}(\bar{p}), \\
\bar{V}_{A}^{\beta} & =\bar{u}\left(\bar{p}_{k}, \lambda_{k}\right)\left(C_{k \tilde{l}}^{\beta, L} P_{L}+C_{k \tilde{l}}^{\beta, R} P_{R}\right) v\left(\bar{p}_{\tilde{l}}, \lambda_{\tilde{l}}\right)=\left[V_{A}^{\beta}(\bar{p})\right]^{*},
\end{aligned}
$$

with $\bar{p}=(E,-\mathbf{p})$. The RHSs of the last two equations give the relations of the CPconjugate vertices $\bar{V}_{A, B}^{\alpha}$ to $V_{A, B}^{\alpha}$. Taking these relationships into account, we can express the squared amplitude of the CP-conjugate process entirely in the $\widetilde{S}_{\alpha}$-space as follows:

$$
\begin{aligned}
\left|\mathcal{T}_{s}^{\bar{A} \rightarrow \bar{B}}(s)\right|^{2} & =\sum_{\lambda_{k}, \lambda_{m}, \lambda_{\tilde{l}}, \lambda_{\tilde{n}}} \bar{V}_{B}^{\dagger} \Delta^{\top} \bar{V}_{A} \bar{V}_{A}^{\dagger}\left(\Delta^{\top}\right)^{\dagger} \bar{V}_{B} \\
& =\operatorname{Tr}\left[T_{B}^{\top}(s) \Delta^{\top}(s) T_{A}^{\top}(s)\left(\Delta^{\top}(s)\right)^{\dagger}\right]=\operatorname{Tr}\left[T_{A}(s) \Delta(s) T_{B}(s) \Delta^{\dagger}(s)\right] .
\end{aligned}
$$

In fact, the last two trace expressions correspond effectively to the discrete transformations CP and T-reversal, respectively. Explicitly, under CP-conjugation, the resummed $\widetilde{S}_{\alpha^{-}}$ propagator matrix and the production and decay matrices transform as

$$
\mathrm{CP}: \Delta(s) \rightarrow \bar{\Delta}(s)=\Delta^{\top}(s) ; \quad T_{A(B)}(s) \rightarrow \bar{T}_{A(B)}(s)=T_{A(B)}^{\top}(s) .
$$

Under T-reversal, we have

$$
\mathrm{T}: \Delta(s) \rightarrow \Delta^{\mathrm{t}}(s)=\Delta(s) ; \quad T_{A, B}(s) \quad \rightarrow \quad T_{A(B)}^{\mathrm{t}}(s)=T_{B(A)}(s) .
$$

Notice that the squared amplitudes (2.13) and (2.17) are manifestly invariant under CPT transformations. 


\section{Unitarity and CPT Constraints on CP Observables}

The unitarity and CPT invariance of the S-matrix introduce non-trivial constraints on CP asymmetries, see e.g. [4. In particular, the equality of the $s$-channel forward amplitude $A \rightarrow A$ with its CPT-conjugate one $\bar{A} \rightarrow \bar{A}$,

$$
\mathcal{T}_{s}^{A \rightarrow A}(s)=\operatorname{Tr}\left[T_{A}(s) \Delta(s)\right]=\mathcal{T}_{s}^{\bar{A} \rightarrow \bar{A}}(s),
$$

gives rise to relations among the different resonant channels in processes, such as $A \rightarrow X$ and its CP-conjugate process $\bar{A} \rightarrow \bar{X}$, where $X(\bar{X})$ is an accessible final state. To make such relations explicit, let us first consider the reactions of $A$ into all possible $X$ states. The sum of the squared amplitudes integrated over the phase space of the $X$ states may be calculated as

$$
\begin{aligned}
\sum_{X} \int \mathrm{dPS}_{X}\left|\mathcal{T}_{s}^{A \rightarrow X}\right|^{2} & =\sum_{X} \operatorname{PS}_{X} \operatorname{Tr}\left[T_{A} \Delta T_{X} \Delta^{\dagger}\right]=-i \operatorname{Tr}\left[T_{A} \Delta\left(\Delta^{-1}-\left(\Delta^{-1}\right)^{\dagger}\right) \Delta^{\dagger}\right] \\
& =i \operatorname{Tr}\left[T_{A}\left(\Delta-\Delta^{\dagger}\right)\right]=-2 \operatorname{Im}\left(\mathcal{T}_{s}^{A \rightarrow A}\right)
\end{aligned}
$$

where we used the relation (2.8) derived from the optical theorem. As a consequence of the CPT constraint (3.1), the total unpolarised cross section of $A$ to all possible $X$ is equal to the corresponding unpolarised cross section of the C-conjugate states $\bar{A}$ to all possible C-conjugate states $\bar{X}$.

Assuming that the dominant source of $\mathrm{CP}$ violation is due to scalar-particle mixing, we define CP-violating observables pertinent to a resonant reaction $A \rightarrow \widetilde{S}_{\alpha} \rightarrow B$ and its CP-conjugate one $\bar{A} \rightarrow \widetilde{S}_{\alpha}^{*} \rightarrow \bar{B}$ :

$$
\Delta_{A \rightarrow B}^{\mathrm{CP}}(s)=\int\left(\left|\mathcal{T}_{s}^{A \rightarrow B}(s)\right|^{2}-\left|\mathcal{T}_{s}^{\bar{A} \rightarrow \bar{B}}(s)\right|^{2}\right) \mathrm{dPS}_{B}
$$

Since we sum over all particle helicities and integrate over the P-invariant phase space, the CP-odd observable $\Delta_{A \rightarrow B}^{\mathrm{CP}}$ is also odd under $\mathrm{C}$, but even under $\mathrm{P}$ transformations.

We will now show that thanks to unitarity and CPT invariance, at least three linearly independent decay channels of $\widetilde{S}_{\alpha}$ are required in $2 \rightarrow 2$ scatterings, in order to obtain non-zero C/CP-odd observables, such as $\Delta_{A \rightarrow B}^{\mathrm{CP}}(s)$ given in (3.3). Our proof for a two-particle scalar mixing system will then be generalized to mixing systems with $n$ scalar particles. In Section 4, we extend this theorem to the correlated production and decay of two unstable-particle systems in $2 \rightarrow 4$ scatterings, where we show that only two independent decay channels are sufficient to obtain a non-zero $\mathrm{C}$ and $\mathrm{CP}$ asymmetry.

\subsection{CP Conditions in Two-Particle-Mixing Transitions}

We will now show that the C- and CP-odd observables $\Delta_{A \rightarrow B}^{\mathrm{CP}}(s)$ defined in (3.3) vanish, if only two independent decay channels are open in $2 \rightarrow 2$ resonant scatterings $A \rightarrow \widetilde{S}_{\alpha} \rightarrow$ $X$. To this end, let us assume that $X=A^{\prime}, B$, where $A^{\prime}$ is the same two-particle state as the initial state $A$ but has different momentum configurations. As a consequence of the CPT-invariance and unitarity relations in (3.1) and (3.2), we have the equality

$$
\int \mathrm{dPS}_{A^{\prime}}\left|\mathcal{T}_{s}^{A \rightarrow A^{\prime}}\right|^{2}+\int \mathrm{dPS}_{B}\left|\mathcal{T}_{s}^{A \rightarrow B}\right|^{2}=\int \mathrm{dPS}_{A^{\prime}}\left|\mathcal{T}_{s}^{\bar{A} \rightarrow \bar{A}^{\prime}}\right|^{2}+\int \mathrm{dPS}_{B}\left|\mathcal{T}_{s}^{\bar{A} \rightarrow \bar{B}}\right|^{2} .
$$


In terms of $\mathrm{CP}$ observables, the above equality can be rewritten as

$$
\begin{aligned}
\Delta_{A \rightarrow B}^{\mathrm{CP}}(s) & =\int \operatorname{dPS}_{B}\left(\left|\mathcal{T}_{s}^{A \rightarrow B}\right|^{2}-\left|\mathcal{T}_{s}^{\bar{A} \rightarrow \bar{B}}\right|^{2}\right)=-\int \operatorname{dPS}_{A^{\prime}}\left(\left|\mathcal{T}_{s}^{A \rightarrow A^{\prime}}\right|^{2}-\left|\mathcal{T}_{s}^{\bar{A} \rightarrow \bar{A}^{\prime}}\right|^{2}\right) \\
& =-\Delta_{A \rightarrow A}^{\mathrm{CP}}(s) .
\end{aligned}
$$

Since the decay channel of $\widetilde{S}_{\alpha}$ into states $A^{\prime}$ is not kinematically independent to the production channel $A \rightarrow \widetilde{S}_{\alpha}$ (they just differ by an overall phase-space factor), we have that $\Delta_{A \rightarrow A}^{\mathrm{CP}}=0$, as a consequence of CPT invariance, implying also that $\Delta_{A \rightarrow B}^{\mathrm{CP}}(s)=0$. Hence, we can conclude that more than two linearly independent decay channels would be needed to obtain non-zero $\mathrm{C}$ and $\mathrm{CP}$ asymmetries in $2 \rightarrow 2$ resonant scatterings.

The above conclusion can also be obtained by an explicit calculation of the squared amplitude $A \rightarrow \widetilde{S}_{\alpha} \rightarrow B$, in a two-particle mixing system; the generalization to a system with $n$ particles follows in the next subsection. Our calculation gets facilitated by a spinorial trace technique, where the $2 \times 2$ production and decay matrices $T_{A, B}$ and the inverse propagator matrix $\Delta^{-1}$ are expanded in terms of the two-by-two unit matrix $\mathbb{1}_{2} \equiv \sigma^{0}$ and the three Pauli matrices $\sigma^{1,2,3}$. Technical details of the trace technique are given in Appendix A. In detail, we obtain for the squared amplitude

$$
\left|\mathcal{T}_{s}^{A \rightarrow B}\right|^{2}=\operatorname{Tr}\left[T_{A} \Delta T_{B} \Delta^{\dagger}\right]=\frac{T_{A}^{\mu} \Delta^{-1, \nu} T_{B}^{\rho}\left(\Delta^{-1, \lambda}\right)^{*}}{\left|\operatorname{det}\left(\Delta^{-1}\right)\right|^{2}} \operatorname{Tr}\left[\sigma_{\mu} \bar{\sigma}_{\nu} \sigma_{\rho} \bar{\sigma}_{\lambda}\right]
$$

where the expansion coefficients are given in Appendix A.2. Since the CP-conjugate amplitude is obtained by the interchange of the Hermitian vertex matrices $T_{A} \leftrightarrow T_{B}$, the only contribution to the CP observable $\Delta_{A \rightarrow B}^{\mathrm{CP}}(s)$ comes from the $\mu \leftrightarrow \rho$ antisymmetric parts of the amplitude squared. These are the terms proportional to the the Levi-Civita tensor $\varepsilon_{\mu \nu \rho \lambda}$ contained in the trace expression over the generalized Pauli spinors on the RHS of (3.6) [cf. (A.10)]. Specifically, up to overall phase-space and other kinematical factors, the $\mathrm{CP}$ observable $\Delta_{A \rightarrow B}^{\mathrm{CP}}(s)$ is

$$
\Delta_{A \rightarrow B}^{\mathrm{CP}}(s) \propto \varepsilon_{\mu \nu \rho \lambda} T_{A}^{\mu} T_{B}^{\nu} \operatorname{Im}\left(\Delta^{-1, \rho}\right) \operatorname{Re}\left(\Delta^{-1, \lambda}\right) .
$$

The 4-vector $\operatorname{Im}\left(\Delta^{-1, \rho}\right)$ derives from absorptive parts of the self-energy,

$$
\operatorname{Im}\left(\Delta^{-1, \rho}\right)=\frac{1}{2} \operatorname{Tr}\left[\bar{\sigma}^{\rho} \Pi^{\mathrm{abs}}\right]=\frac{1}{2}\left(\mathrm{PS}_{A} T_{A}^{\rho}+\mathrm{PS}_{B} T_{B}^{\rho}\right)
$$

where $\mathrm{PS}_{A, B}$ denote phase-space factors associated with the $A$ and $B$ states. Evidently, three linearly independent channels or 4 -vectors in the generalized Pauli-spinor space are needed, e.g. $T_{A, B, C}^{\rho}$, in order to obtain a non-zero CP asymmetry $\Delta_{A \rightarrow B}^{\mathrm{CP}}$ in (3.7).

\subsection{Beyond the Two-Particle Mixing}

The above result obtained for a two-particle-mixing system can now be generalized to mixing systems with $n$ unstable particles $\widetilde{S}_{\alpha}$, with $\alpha=1,2, \ldots, n$.

Our starting point is the $n \times n$ propagator matrix $\Delta$ given by

$$
\Delta(s)=\left[\mathrm{D}^{-1}(s)+i \Pi(s)\right]^{-1} .
$$

The $n \times n$ propagator matrix $\Delta(s)$ consists of a Hermitian matrix $\mathrm{D}_{\alpha \beta}^{-1}(s)=\delta_{\alpha \beta}(s-$ $\left.M_{\alpha}^{2}\right)+\Pi_{\alpha \beta}^{\text {disp }}(s)$, which may include dispersive contributions, and a general anti-Hermitian 
matrix $i \Pi_{\alpha \beta}(s)=i \prod_{\alpha \beta}^{\text {abs }}(s)$ that describes the absorptive effects. Dropping the explicit $s$-dependence, it can be rewritten in the more convenient form:

$$
\Delta=(\mathrm{D}-i \mathrm{D} \Pi \mathrm{D})\left[\mathbb{1}+(\Pi \mathrm{D})^{2}\right]^{-1}=\left[\mathbb{1}+(\mathrm{D} \Pi)^{2}\right]^{-1}(\mathrm{D}-i \mathrm{D} \Pi \mathrm{D}),
$$

where $\mathbb{1}$ is the $n$-dimensional unit matrix.

The cross sections for the $2 \rightarrow 2$ resonant process $A \rightarrow \widetilde{S}_{\alpha} \rightarrow B$ and its CP-conjugate one $\bar{A} \rightarrow \widetilde{S}_{\alpha}^{*} \rightarrow \bar{B}$ may straightforwardly be evaluated by the trace expressions

$$
\sigma=\operatorname{Tr}\left[T_{B} \Delta T_{A} \Delta^{\dagger}\right], \quad \sigma^{\mathrm{CP}}=\operatorname{Tr}\left[T_{A} \Delta T_{B} \Delta^{\dagger}\right]
$$

where phase-space integration is implied. From these, the CP-violating difference of the two cross sections, $\Delta \sigma^{\mathrm{CP}} \equiv \sigma-\sigma^{\mathrm{CP}}$, can be defined, which is calculated as

$$
\begin{aligned}
\Delta \sigma^{\mathrm{CP}}= & \frac{1}{2} \operatorname{Tr}\left[T_{B}\left(\Delta-\Delta^{\dagger}\right) T_{A}\left(\Delta+\Delta^{\dagger}\right)-T_{B}\left(\Delta+\Delta^{\dagger}\right) T_{A}\left(\Delta-\Delta^{\dagger}\right)\right] \\
= & -2 i \operatorname{Tr}\left\{T_{B} \mathrm{D} \Pi\left[\mathbb{1}+(\mathrm{D} \Pi)^{2}\right]^{-1} \mathrm{D} T_{A}\left[\mathbb{1}+(\mathrm{D} \Pi)^{2}\right]^{-1} \mathrm{D}\right. \\
& \left.\quad-T_{B}\left[\mathbb{1}+(\mathrm{D} \Pi)^{2}\right]^{-1} \mathrm{D} T_{A} \mathrm{D} \Pi\left[\mathbb{1}+(\mathrm{D} \Pi)^{2}\right]^{-1} \mathrm{D}\right\} .
\end{aligned}
$$

From the last expression in (3.12), we observe that the CP-violating difference $\Delta \sigma^{\mathrm{CP}}$ can be cast into the compact form:

$$
\Delta \sigma^{\mathrm{CP}}=-2 i \operatorname{Tr}\left\{\mathrm{D} \Pi\left[\left(\mathbb{1}+(\mathrm{D} \Pi)^{2}\right)^{-1} \mathrm{D} T_{A},\left(\mathbb{1}+(\mathrm{D} \Pi)^{2}\right)^{-1} \mathrm{D} T_{B}\right]\right\}
$$

where $[X, Y] \equiv X Y-Y X$ is the commutator for two $n \times n$ matrices $X$ and $Y$. Clearly, if $T_{B}=c T_{A}$, with $c \in \mathbb{C}$, the commutator in (3.13) vanishes. Further, from the cyclicity of the trace,

$$
\operatorname{Tr}\{A[B, C]\}=\operatorname{Tr}\{C[A, B]\}=\operatorname{Tr}\{B[C, A]\},
$$

we have that the $\mathrm{CP}$-violating difference vanishes, i.e. $\Delta \sigma^{\mathrm{CP}}=0$, if

$$
\left[\left(\mathbb{1}+(\mathrm{D} \Pi)^{2}\right)^{-1} \mathrm{D} T_{A}, \mathrm{D} \Pi\right]=0
$$

or if

$$
\left[\left(\mathbb{1}+(\mathrm{D} \Pi)^{2}\right)^{-1} \mathrm{D} T_{B}, \mathrm{D} \Pi\right]=0
$$

Since the absorptive contributions can in general be parametrized by the different open decay channels, $\Pi=\alpha T_{A}+\beta T_{B}+\gamma T_{C}$, then $\Delta \sigma^{\mathrm{CP}}$ will vanish if $T_{C}=0$ (only 2 open decay channels), or if $T_{C}=c_{1} T_{A}+c_{2} T_{B}$ is linearly dependent. Consequently, at least three linearly independent decay channels are needed to construct non-vanishing CP asymmetries in general $2 \rightarrow 2$ scattering processes. As we will see in the next section, the validity of this theorem may be extended to unstable states which are pair produced and decay in correlation in $2 \rightarrow 4$ scattering reactions, where however only two independent channels are needed.

We conclude this section by remarking that in the absence of a particle mixing, the decay matrices $T_{A, B, C}$ and $\Pi$ are diagonal, and the commutator in (3.13) vanishes, 


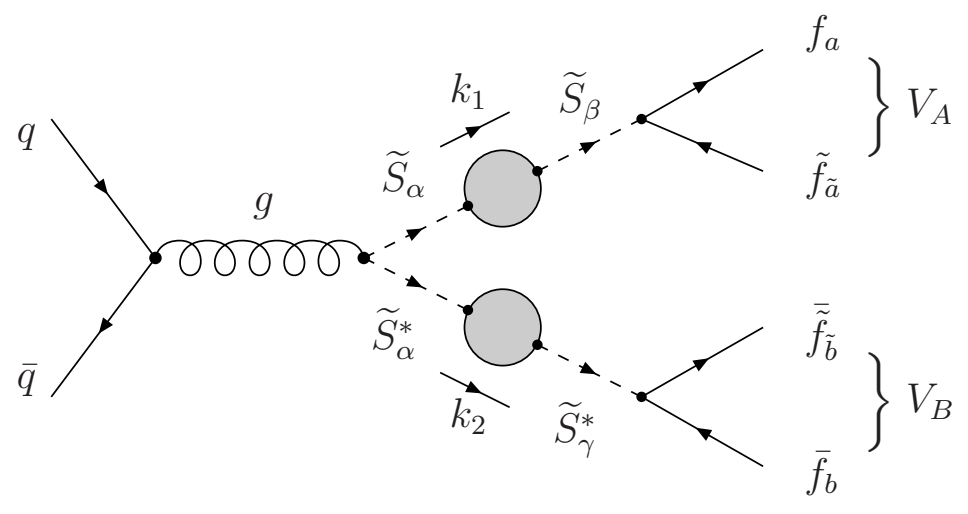

Figure 3: Correlated production and decay of $\widetilde{S}_{\alpha}$ particles in $2 \rightarrow 4$ scatterings at highenergy hadron colliders.

leading to $\Delta \sigma^{\mathrm{CP}}=0$. This corresponds to the case where each unstable particle couples to a disjoint set of states. This situation can happen naturally, only if the unstable particles carry their own conserved charges. In minimal supersymmetric theories, the mixing of light squarks or sleptons is suppressed by their masses, such that CP asymmetries are sizable, only if particular resonant conditions are met [4,12,13. As we will see in the next section, we find sizable CP asymmetries in the strongly mixed stop or sbottom sectors for a wide range of MSSM parameters.

\section{EPR Correlated Production and Decay of Scalars}

In this section we study the EPR-correlated production and decay of unstable scalar particles at high-energy colliders. Specifically, we consider the resonant part of $2 \rightarrow 4$ processes, mediated by coloured or charged scalar particles $\widetilde{S}_{\alpha}$, as displayed in Fig. 3 ,

The transition amplitude for the $2 \rightarrow 4$ partonic process $q \bar{q} \rightarrow \widetilde{S}_{\alpha} \widetilde{S}_{\alpha}^{*} \rightarrow(f \tilde{f})\left(\bar{f}^{\prime} \overline{\tilde{f}}^{\prime}\right)$ has the analytical form

$$
\mathcal{T}_{s}^{2 \rightarrow 4}\left(k_{1}, k_{2}\right)=J_{g}\left(k_{1}, k_{2}\right) V_{A}^{\dagger}\left(k_{1}\right) \Delta\left(s_{1}\right) \Delta\left(s_{2}\right) V_{B}\left(k_{2}\right),
$$

where the initial gluon current factorizes in the amplitude, $J_{g}\left(k_{1}, k_{2}\right)=J_{g}^{\mu}\left(k_{1}-k_{2}\right)_{\mu} 3$. Absorbing all kinematical factors into an overall normalization constant $\mathcal{N}$, the cross section for the $2 \rightarrow 4$ scattering is given by

$$
\sigma^{2 \rightarrow 4}(s)=\mathcal{N} \operatorname{Tr}\left[T_{A}\left(s_{1}\right) \Delta\left(s_{1}\right) \Delta\left(s_{2}\right) T_{B}\left(s_{2}\right) \Delta^{\dagger}\left(s_{2}\right) \Delta^{\dagger}\left(s_{1}\right)\right],
$$

where $s=\left(k_{1}+k_{2}\right)^{2} \gg M_{\alpha}^{2}, s_{1,2}=k_{1,2}^{2}$ and phase-space integration is understood.

Since we are interested in the dominant part of the cross section, we expand $\sigma^{2 \rightarrow 4}(s)$ about the resonant region $\sqrt{\bar{s}}=\left(M_{1}+M_{2}\right) / 2$. Hence, we obtain for the resonant (res) part of the cross section

$$
\sigma_{\text {res }}^{2 \rightarrow 4}(s)=\mathcal{N} \operatorname{Tr}\left[T_{A}(\bar{s})(\Delta(\bar{s}))^{2} T_{B}(\bar{s})\left(\Delta^{\dagger}(\bar{s})\right)^{2}\right]
$$

\footnotetext{
${ }^{3}$ Here we discuss the LHC suppressed, but more intuitive one-gluon exchange process. Other processes, such as $g g \rightarrow \widetilde{S}_{\alpha} \widetilde{S}_{\alpha}^{*} \rightarrow(f \tilde{f})\left(\bar{f}^{\prime} \overline{\tilde{f}}^{\prime}\right)$, exhibit similar analytical features and can be treated in analogous fashion.
} 
where we have ignored sub-dominant terms proportional to $\left(s_{1}-s_{2}\right) / \bar{s}$.

To linearize the expression (4.3) in terms of the propagator matrix $\Delta(\bar{s})$ and its Hermitian conjugate $\Delta^{\dagger}(\bar{s})$, we define

$$
\Delta(x ; s) \equiv\left[x \mathbb{1}-M_{\alpha}^{2}+i \Pi_{\alpha \beta}^{\mathrm{abs}}(s)\right]^{-1},
$$

with $\Delta(s ; s)=\Delta(s)$, such that

$$
(\Delta(s))^{2}=(\Delta(s ; s))^{2}=-\left.\frac{\partial}{\partial x} \Delta(x ; s)\right|_{x=s} .
$$

With the help of this last relation, the resonant part of the $2 \rightarrow 4$ cross section can now be recast into the form:

$$
\sigma_{\text {res }}^{2 \rightarrow 4}=\left.\mathcal{N} \frac{\partial^{2}}{\partial x \partial y} \operatorname{Tr}\left[T_{A}(\bar{s}) \Delta(x ; \bar{s}) T_{B}(\bar{s}) \Delta^{\dagger}(y ; \bar{s})\right]\right|_{x=y=\bar{s}} .
$$

Note that this form is very similar to the squared amplitude of a $2 \rightarrow 2$ resonant process [cf. (3.6)]. For the specific two particle mixing case, the resonant part of the $2 \rightarrow 4$ cross section is given by

$$
\sigma_{\text {res }}^{2 \rightarrow 4}=\left.\mathcal{N} \frac{\partial^{2}}{\partial x \partial y} \frac{\Delta^{-1, \nu}(x ; \bar{s})\left(\Delta^{-1, \lambda}(y ; \bar{s})\right)^{*}}{\operatorname{det}\left[\Delta^{-1}(x ; \bar{s})\right] \operatorname{det}\left[\Delta^{-1}(y ; \bar{s})\right]^{*}}\right|_{x=y=\bar{s}} \times T_{A}^{\mu} T_{B}^{\rho} \operatorname{Tr}\left[\sigma_{\mu} \bar{\sigma}_{\nu} \sigma_{\rho} \bar{\sigma}_{\lambda}\right] .
$$

From this last expression, we may calculate the CP-odd part of the $2 \rightarrow 4$ cross section, which may conveniently be written down as

$$
\left(\sigma_{\text {res }}^{2 \rightarrow 4}\right)^{Q \mathrm{P}}=4 \mathcal{N} \varepsilon_{\mu \nu \rho \lambda} T_{A}^{\mu} T_{B}^{\nu} \operatorname{Im}\left(\frac{\partial}{\partial x} \frac{\Delta^{-1, \rho}(x ; \bar{s})}{\operatorname{det}\left[\Delta^{-1}(x ; \bar{s})\right]}\right) \operatorname{Re}\left(\frac{\partial}{\partial x} \frac{\Delta^{-1, \lambda}(x ; \bar{s})}{\operatorname{det}\left[\Delta^{-1}(x ; \bar{s})\right]}\right)
$$

where $x$ is set to $\bar{s}$ after differentiation. Unlike in the $2 \rightarrow 2$ scatterings [cf. (3.7)], we may convince ourselves that for $2 \rightarrow 4$ scatterings, only two linearly independent decay channels would be sufficient, i.e.

$$
\operatorname{Im}\left(\Delta^{-1, \rho}(\bar{s})\right)=\alpha T_{A}^{\rho}+\beta T_{B}^{\rho}
$$

in order to obtain a non-zero CP-odd contribution to the cross section. Specifically, it is not difficult to see that the CP-odd part $\left(\sigma_{\text {res }}^{2 \rightarrow 4}\right)^{Q \mathrm{P}}$ contains terms proportional to

$$
\left.\varepsilon_{\mu \nu \rho \lambda} T_{A}^{\mu} T_{B}^{\nu} \frac{\partial \operatorname{Re} \Delta^{-1, \rho}(x ; \bar{s})}{\partial x}\right|_{x=\bar{s}} \operatorname{Re} \Delta^{-1, \lambda}(\bar{s}) \operatorname{Im}(\operatorname{det}[\Delta(\bar{s})]),
$$

which are clearly non-zero, if only two channels as given in (4.9) are assumed.

In our discussion, we have ignored other one-loop effects, such as gluon-scalar-scalar vertex corrections. These corrections are sub-dominant, but become relevant in restoring the gauge-fixing-parameter independence of the gluon propagator by means of WardTakahashi identities (for a recent review, see [14]). In particular, in the Feynman gauge, one can show that all these effects are non-resonant and can thus be safely neglected. A detailed study of these higher-order effects is beyond the scope of the present paper. 


\subsection{Application to Stop CP Violation at the LHC}

As an application of our results, we consider the pair production and decay of supersymmetric scalar top (or bottom) quarks [6]. These coloured particles can be copiously produced via partonic QCD interactions at hadron colliders. Their sizable mixing can lead to large asymmetries, due to non-vanishing SUSY CP phases in the heavy squark sector $\tilde{q}, q=t, b$. Their dominant decay modes are

$$
\begin{aligned}
& \tilde{q}_{1,2} \rightarrow q+\tilde{g}, \\
& \tilde{q}_{1,2} \rightarrow q+\tilde{\chi}_{1,2,3,4}^{0}, \\
& \tilde{q}_{1,2} \rightarrow q^{\prime}+\tilde{\chi}_{1,2}^{+} .
\end{aligned}
$$

Instead, the decays of $\tilde{q}_{1,2}$ into neutral bosons are closed for sufficiently small $\tilde{q}_{1}-\tilde{q}_{2}$ mass splittings, i.e.

$$
\begin{aligned}
& \tilde{q}_{2} \nrightarrow \tilde{q}_{1}+H_{1,2,3}^{0}, \\
& \tilde{q}_{2} \nrightarrow \tilde{q}_{1}+Z^{0} .
\end{aligned}
$$

Likewise, their decays into charged bosons are closed for sufficiently small stop and sbottom mass differences, i.e.

$$
\begin{array}{lll}
\tilde{q}_{1,2} & \nrightarrow & \tilde{q}_{1,2}^{\prime}+H^{+}, \\
\tilde{q}_{1,2} & \nrightarrow & \tilde{q}_{1,2}^{\prime}+W^{+} .
\end{array}
$$

Stop (and sbottom) decays at the one-loop level within the CP-violating MSSM have been calculated in [7], and in the CP-conserving MSSM in [8].

For our numerical example, we choose the MSSM parameters $M_{\tilde{Q}}=M_{\tilde{U}}=M_{\tilde{D}}=$ $470 \mathrm{GeV}, M_{\tilde{L}}=M_{\tilde{E}}=120 \mathrm{GeV},\left|A_{t}\right|=\left|A_{b}\right|=\left|A_{\tau}\right|=110 \mathrm{GeV}, M_{2}=2 M_{1}=|\mu|=$ $250 \mathrm{GeV}, m_{\tilde{g}}=710 \mathrm{GeV}, \tan \beta=5$, and the phases $\phi_{A_{t}}=\pi / 4, \phi_{A_{b}}=\phi_{A_{\tau}}=\phi_{M_{1}}=$ $\phi_{\mu}=\phi_{\tilde{g}}=0$. At tree level, the resulting stop masses, widths, and mixing angle are $m_{\tilde{t}_{\{1,2\}}}=\{485,514\} \mathrm{GeV}, \Gamma_{\tilde{t}_{\{1,2\}}}=\{5.3,9.1\} \mathrm{GeV}, \cos \theta_{\tilde{t}}=-0.725$. The gaugino masses are $m_{\tilde{\chi}_{\{1,2\}}^{ \pm}}=\{187,321\} \mathrm{GeV}, m_{\tilde{\chi}_{\{1,2,3,4\}}^{0}}=\{115,194,256,322\} \mathrm{GeV}$.

As an illustrative example, we study stop pair production via the process $q \bar{q} \rightarrow$ $\tilde{t}_{\alpha} \tilde{t}_{\alpha}^{*}$, decaying into charginos: $\tilde{t}_{\alpha} \rightarrow b \tilde{\chi}_{1}^{+}(A), \tilde{t}_{\alpha}^{*} \rightarrow \bar{b} \tilde{\chi}_{2}^{-}(B)$. At the resonant region, $\sqrt{\bar{s}}=\left(m_{\tilde{t}_{1}}+m_{\tilde{t}_{2}}\right) / 2$, the transition amplitude squared and its CP-conjugate one follow from (4.3) to be

$$
\begin{aligned}
|\mathcal{T}(\bar{s})|^{2} & =\operatorname{Tr}\left[T_{A}(\bar{s})(\Delta(\bar{s}))^{2} T_{B}(\bar{s})\left(\Delta^{\dagger}(\bar{s})\right)^{2}\right], \\
|\overline{\mathcal{T}}(\bar{s})|^{2} & =\operatorname{Tr}\left[T_{B}(\bar{s})(\Delta(\bar{s}))^{2} T_{A}(\bar{s})\left(\Delta^{\dagger}(\bar{s})\right)^{2}\right] .
\end{aligned}
$$

In Fig. 4, we show the phase $\phi_{A_{t}}$ and the stop mass dependence of the CP asymmetry

$$
\mathcal{A}=\frac{|\mathcal{T}|^{2}-|\overline{\mathcal{T}}|^{2}}{|\mathcal{T}|^{2}+|\overline{\mathcal{T}}|^{2}}
$$

We observe that the $\mathrm{CP}$ asymmetry can reach values of order one, thanks to a maximal mixing of the two stop states in production and decay. The CP asymmetry would decrease as stop mass splitting increases, which can occur for large mass differences $M_{\tilde{Q}}-M_{\tilde{U}}$. 


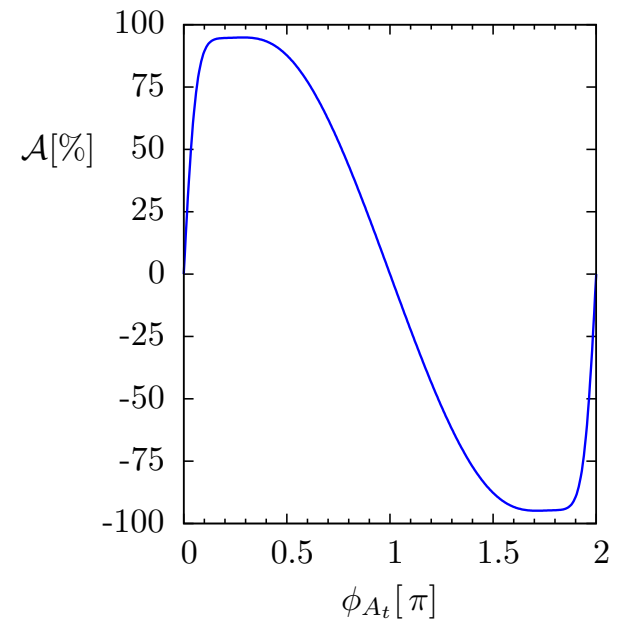

(a)

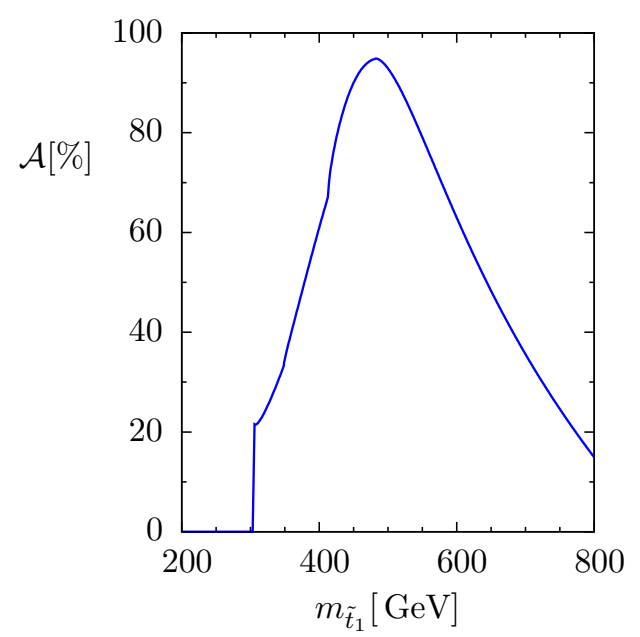

(c)

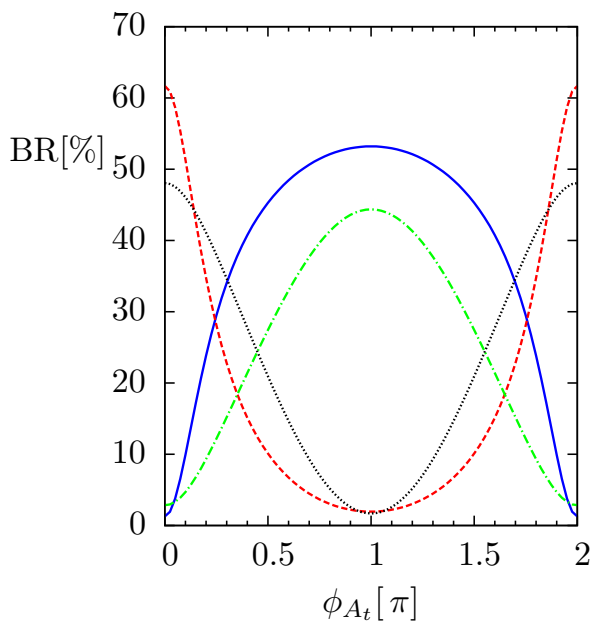

(b)

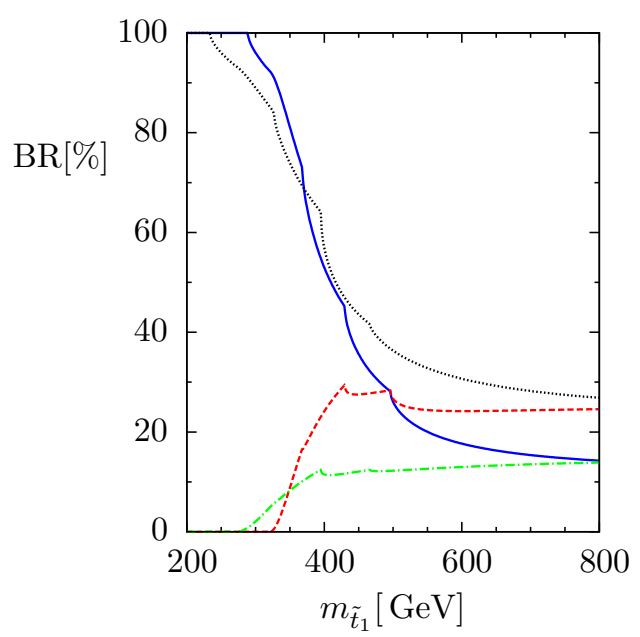

(d)

Figure 4: The CP asymmetry $\mathcal{A}$, see (4.20), for stop pair production $q \bar{q} \rightarrow \tilde{t}_{\alpha}(\bar{s}) \tilde{t}_{\alpha}^{*}(\bar{s})$ and decay $\tilde{t}_{\alpha} \rightarrow b \tilde{\chi}_{1}^{+}, \tilde{t}_{\alpha}^{*} \rightarrow \bar{b} \tilde{\chi}_{2}^{-}$, at $\sqrt{\bar{s}}=\left(m_{\tilde{t}_{1}}+m_{\tilde{t}_{2}}\right) / 2$, as functions of $\phi_{A_{t}}$ and $m_{\tilde{t}_{1}}$. Also shown are the branching ratios $\operatorname{BR}\left(\tilde{t}_{1} \rightarrow b \tilde{\chi}_{1}^{+}\right)$(solid, blue), $\operatorname{BR}\left(\tilde{t}_{1} \rightarrow b \tilde{\chi}_{2}^{+}\right)$(dashed, red), $\operatorname{BR}\left(\tilde{t}_{2} \rightarrow b \tilde{\chi}_{1}^{+}\right)$(dotted, black), $\operatorname{BR}\left(\tilde{t}_{2} \rightarrow b \tilde{\chi}_{2}^{+}\right)$(dash-dotted, green), as functions of $\phi_{A_{t}}$ and $m_{\tilde{t}_{1}}$. In the $m_{\tilde{t}_{1}}$-plots (c) and (d), $M_{\tilde{Q}}=M_{\tilde{U}}=M_{\tilde{D}}$ is varied, whilst the other MSSM parameters are fixed as given in the text of subsection 4.1 .

In Fig. 5, we study in more detail the dependence of the amplitudes [cf. (4.2)],

$$
\begin{aligned}
\left|\mathcal{T}\left(s_{1}, s_{2}\right)\right|^{2} & =\operatorname{Tr}\left[T_{A}\left(s_{1}\right) \Delta\left(s_{1}\right) \Delta\left(s_{2}\right) T_{B}\left(s_{2}\right) \Delta^{\dagger}\left(s_{2}\right) \Delta^{\dagger}\left(s_{1}\right)\right] \\
\left|\overline{\mathcal{T}}\left(s_{1}, s_{2}\right)\right|^{2} & =\operatorname{Tr}\left[T_{B}\left(s_{1}\right) \Delta\left(s_{1}\right) \Delta\left(s_{2}\right) T_{A}\left(s_{2}\right) \Delta^{\dagger}\left(s_{2}\right) \Delta^{\dagger}\left(s_{1}\right)\right]
\end{aligned}
$$

and the corresponding CP asymmetry as defined in (4.20), as functions of $\delta \sqrt{s}=\left(\sqrt{s_{1}}-\right.$ $\left.\sqrt{s_{2}}\right) / 2$. In our numerical estimates, we use the parameterization

$$
\sqrt{s_{1}}=\sqrt{\bar{s}}+\delta \sqrt{s}, \quad \sqrt{s_{2}}=\sqrt{\bar{s}}-\delta \sqrt{s},
$$




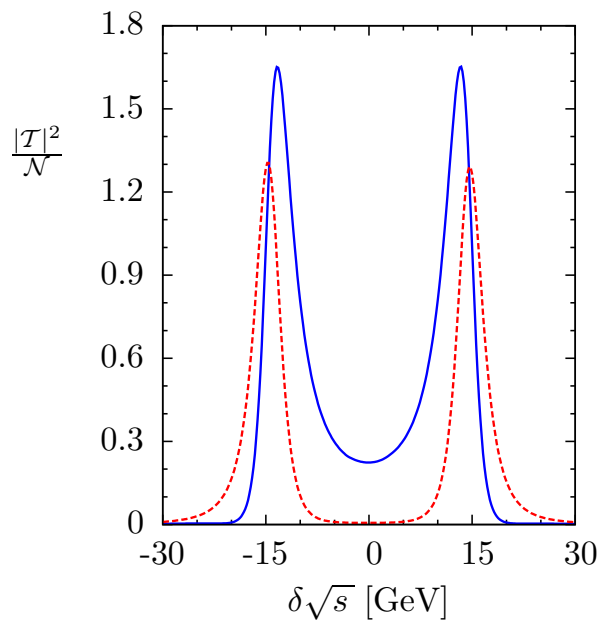

(a)

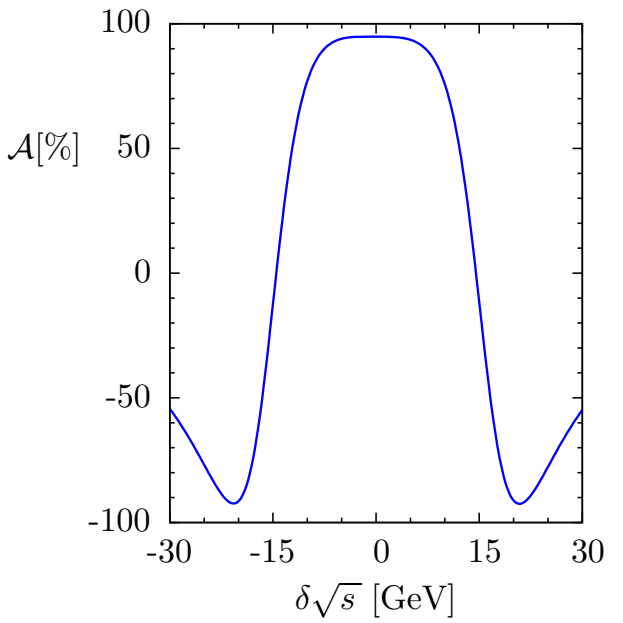

(b)

Figure 5: The dependence on $\delta \sqrt{s}=\left(\sqrt{s_{1}}-\sqrt{s_{2}}\right) / 2$ of (a) the amplitude squared $\left|\mathcal{T}\left(s_{1}, s_{2}\right)\right|^{2}$ 4.21) (up to an arbitrary normalization $\mathcal{N}$ ), with $\sqrt{s_{1,2}}=\sqrt{\bar{s}} \pm \delta \sqrt{s}$ at the resonance region $\sqrt{\bar{s}}=\left(m_{\tilde{t}_{1}}+m_{\tilde{t}_{2}}\right) / 2$, for stop pair production $q \bar{q} \rightarrow \tilde{t}_{\alpha}\left(s_{1}\right) \tilde{t}_{\alpha}^{*}\left(s_{2}\right)$ and decay $\tilde{t}_{\alpha} \rightarrow b \tilde{\chi}_{1}^{+}, \tilde{t}_{\alpha}^{*} \rightarrow \bar{b} \tilde{\chi}_{2}^{-}$(solid, blue), and the CP-conjugate amplitude squared (4.22) for the process $\tilde{t}_{\alpha} \rightarrow b \tilde{\chi}_{2}^{+}, \tilde{t}_{\alpha}^{*} \rightarrow \bar{b} \tilde{\chi}_{1}^{-}$(dashed, red), and (b) the corresponding CP asymmetry (4.20), with MSSM parameters as given in the text of subsection 4.1.

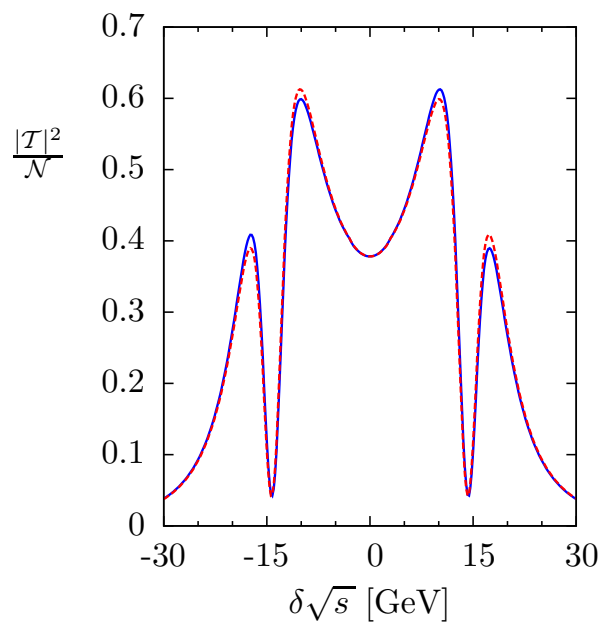

(a)

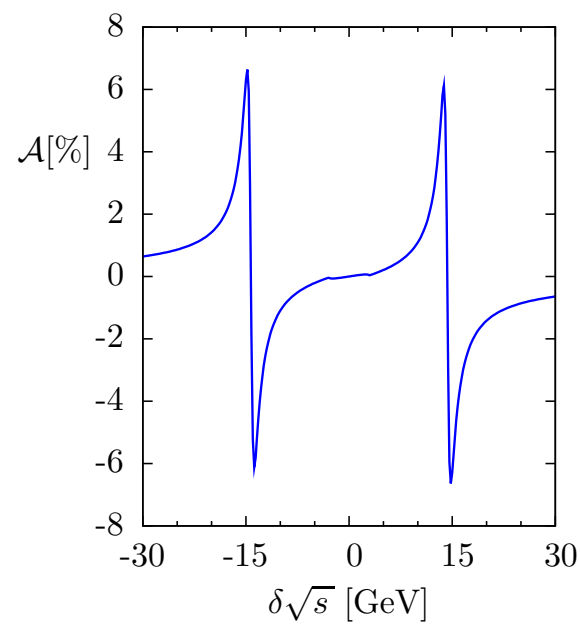

(b)

Figure 6: The same as in Fig. 5 for (a) the stop pair production $q \bar{q} \rightarrow \tilde{t}_{\alpha}\left(s_{1}\right) \tilde{t}_{\alpha}^{*}\left(s_{2}\right)$ and decay $\tilde{t}_{\alpha} \rightarrow b \tilde{\chi}_{1}^{+}, \tilde{t}_{\alpha}^{*} \rightarrow \bar{b} \tilde{\chi}_{1}^{-}$(solid, blue), and the CP-conjugate amplitude squared for the process $\tilde{t}_{\alpha} \rightarrow b \tilde{\chi}_{1}^{+}, \tilde{t}_{\alpha}^{*} \rightarrow \bar{b} \tilde{\chi}_{1}^{-}$(dashed, red), and for (b) the corresponding CP asymmetry.

around the resonance region $\sqrt{\bar{s}}=\left(m_{\tilde{t}_{1}}+m_{\tilde{t}_{2}}\right) / 2$. As can be seen from Fig. 5 , the CP asymmetry $\mathcal{A}$ can be of order 1 for the experimentally testable interval of $\delta \sqrt{s} \sim 20 \mathrm{GeV}$.

Finally, in Fig. 6 we display numerical estimates of stop decays into the same states: $\tilde{t}_{\alpha} \rightarrow b \tilde{\chi}_{1}^{+}(A), \tilde{t}_{\alpha}^{*} \rightarrow \bar{b} \tilde{\chi}_{1}^{-}(\bar{A})$. The corresponding CP asymmetry $\mathcal{A}$ is non-vanishing, of order $5 \%$, only for non-zero values of $\delta \sqrt{s}$. Hence, CP violation in the same channel would be more difficult to probe for the stop scenario of the MSSM under study. 
Before closing this section, it is interesting to comment on the discovery potential of the LHC in measuring a non-zero CP asymmetry. As shown in [15], the minimal luminosity $\mathcal{L}$ to observe a non-vanishing $\mathrm{CP}$ asymmetry $\mathcal{A}$ may be estimated by

$$
\mathcal{L}=\frac{n^{2}}{\sigma}\left(\frac{1}{\mathcal{A}^{2}}-1\right)
$$

where $n$ is the number of standard deviations (confidence level) and $\sigma$ is the cross section of the signal events. Obviously, to obtain realistic values of statistical significances and luminosities requires detailed Monte-Carlo simulations. Since such a study is beyond the scope of the present paper, here we only offer an initial estimate.

For our benchmark scenario, with $m_{\tilde{t}_{1,2}} \approx 500 \mathrm{GeV}$, the cross section for stop pair production is $\sigma_{p}\left(p p \rightarrow \tilde{t}_{\alpha} \tilde{t}_{\alpha}\right) \approx \mathcal{O}(700) \mathrm{fb}$, for $\alpha=1$, 2, at the next-to-leading order, according to the public code PROSPINO [16. The stop branching ratios into charginos are found approximately to be $\operatorname{BR}\left(\tilde{t}_{\alpha} \rightarrow b \tilde{\chi}_{j}^{ \pm}\right) \approx 20 \%$, for each $j=1,2$ and $\alpha=1,2$ (see panels (b) and (d) in Fig. (4). Correspondingly, the leptonic $(\ell=e, \mu)$ branching ratios of the charginos are $\operatorname{BR}\left(\tilde{\chi}_{j}^{ \pm} \rightarrow \tilde{\nu}_{\ell} \ell\right) \approx 30 \%$, using the formulae quoted in [17] and the fact that the sneutrinos $\tilde{\nu}_{\ell}$ decay invisibly in our scenario, i.e. $\tilde{\nu}_{\ell} \rightarrow \nu_{\ell} \tilde{\chi}_{1}^{0}$.

Taking all the above estimates into account, we find a net signal cross section $\sigma \approx$ $\mathcal{O}(3) \mathrm{fb}$, for stop pair production and decay. In addition to using standard cuts to reduce the background and isolate the signal, one has still to identify the charges of the final leptons and the mother chargino states, $\tilde{\chi}_{1}^{ \pm}$or $\tilde{\chi}_{2}^{ \pm}$, for a proper measurement of the CP asymmetries. Recently, a similar study of triple-product asymmetries in stop pairproduction and decay [18] has shown that the method of kinematic event selection allows such an identification of the decay chains, if the particle masses are known, presumably from other measurements. Because of the kinematic cuts and the event selection criteria, the signal events are reduced typically by an order of magnitude, with the surviving CP-even background being similar in size to the signal [18. Based on these results, our CP-asymmetry is expected to be reduced by half, such that we are left with $\mathcal{A} \approx 50 \%$ in the best case scenario. Inserting these numbers into Eq. (4.24), we find $\mathcal{L} \approx n^{2} 10 \mathrm{fb}^{-1}$. Thus, LHC luminosities of order a few $10 \mathrm{fb}^{-1}$ would be sufficient to observe resonant EPR CP asymmetries of order one. 


\section{Conclusions}

We have analyzed resonant CP-violating Einstein-Podolsky-Rosen (EPR) correlations in the pair production and decay of unstable scalar particles at high-energy colliders. We have shown that as a consequence of unitarity and CPT invariance of the S-matrix, a minimum number of linearly independent decay matrices associated with the unstable scalar states are necessary in order to obtain a non-vanishing result for CP-odd observables that are also odd under C-conjugation, but even under $\mathrm{P}$ transformations. For $2 \rightarrow 2$ scatterings, at least three such independent decay matrices are needed, whereas for $2 \rightarrow 4$ scatterings, only two independent decay matrices are required. Even though our study involved the mixing of scalar particles only, we may safely conjecture that our results will also hold true for the mixing of unstable fermions and vector bosons.

As a direct application of the above theorem, we have presented numerical estimates of CP asymmetries for the correlated production and decay of supersymmetric scalar topanti-top pairs at the LHC. We have explicitly demonstrated that the CP asymmetries could be sizeable, reaching values of order one and so making them directly testable. Our analysis also led us to the development a novel spinorial trace formalism, which helped us to efficiently evaluate lengthy expressions of squared amplitudes describing resonant scalar transitions. The formalism presented here could be extended to other unstable-particle systems, predicted in minimal extensions of the Standard Model that include mixing of Higgs-boson states [19,20] or the mixing of heavy unstable neutrinos [21]. It is interesting to study systematically possible phenomena of resonant CP-violating EPR correlations in these systems both for the LHC and future high-energy colliders.

\section{Acknowledgments}

We thank Jose Bernabéu, Peter Millington and Federico v. d. Pahlen for useful discussions. OK gratefully acknowledges partial support by MICINN project FPA.2006-05294 and CPAN, and AP the STFC research grant, ref: ST/J000418/1. 


\section{A Spinorial Trace Technique for Two Scalar Mixing}

Here we present our conventions for our spinorial trace technique, as well as useful identities and analytical expressions for the case of the two-unstable-particle mixing.

\section{A.1 Preliminaries}

A basic element of our formalism are the generalized Pauli matrices [6]:

$$
\sigma^{\mu}=\left(\sigma^{0}, \boldsymbol{\sigma}\right), \quad \bar{\sigma}^{\mu}=\left(\sigma^{0},-\boldsymbol{\sigma}\right)
$$

with

$$
\sigma^{0}=\left(\begin{array}{ll}
1 & 0 \\
0 & 1
\end{array}\right), \quad \sigma^{1}=\left(\begin{array}{ll}
0 & 1 \\
1 & 0
\end{array}\right), \quad \sigma^{2}=\left(\begin{array}{cc}
0 & -i \\
i & 0
\end{array}\right), \quad \sigma^{3}=\left(\begin{array}{cc}
1 & 0 \\
0 & -1
\end{array}\right) .
$$

Note that these are also the generators of the $\mathrm{SL}(2, \mathbb{C})$ group. Then, any $2 \times 2$-dimensional complex matrix $A$ can be expanded as

$$
A=A_{\mu} \sigma^{\mu}=A^{\mu} \bar{\sigma}_{\mu}, \quad A^{-1}=\frac{A_{\mu} \bar{\sigma}^{\mu}}{A_{\nu} A^{\nu}}
$$

where $A_{\nu} A^{\nu}=\operatorname{det} A$ and the complex components $A^{\nu}$ may be evaluated as

$$
A^{\nu}=\frac{1}{2} \operatorname{Tr}\left[A \bar{\sigma}^{\nu}\right] .
$$

In calculating traces, the following relations are useful [6]:

$$
\begin{aligned}
\sigma^{\mu} \bar{\sigma}^{\nu} \sigma^{\rho}+\sigma^{\rho} \bar{\sigma}^{\nu} \sigma^{\mu} & =2\left(g^{\mu \nu} \sigma^{\rho}-g^{\mu \rho} \sigma^{\nu}+g^{\nu \rho} \sigma^{\mu}\right), \\
\sigma^{\mu} \bar{\sigma}^{\nu} \sigma^{\rho}-\sigma^{\rho} \bar{\sigma}^{\nu} \sigma^{\mu} & =-2 i \varepsilon^{\mu \nu \rho \lambda} \sigma^{\lambda}, \\
\bar{\sigma}^{\mu} \sigma^{\nu} \bar{\sigma}^{\rho}+\bar{\sigma}^{\rho} \sigma^{\nu} \bar{\sigma}^{\mu} & =2\left(g^{\mu \nu} \bar{\sigma}^{\rho}-g^{\mu \rho} \bar{\sigma}^{\nu}+g^{\nu \rho} \bar{\sigma}^{\mu}\right), \\
\bar{\sigma}^{\mu} \sigma^{\nu} \bar{\sigma}^{\rho}-\bar{\sigma}^{\rho} \sigma^{\nu} \bar{\sigma}^{\mu} & =2 i \varepsilon^{\mu \nu \rho \lambda} \bar{\sigma}^{\lambda},
\end{aligned}
$$

with the convention $\varepsilon_{0123}=+1$. For instance, the traces involving two and four generalized Pauli matrices are given by

$$
\begin{aligned}
\operatorname{Tr}\left[\sigma^{\mu} \bar{\sigma}^{\nu}\right] & =2 g^{\mu \nu}, \\
\operatorname{Tr}\left[\sigma^{\mu} \bar{\sigma}^{\nu} \sigma^{\rho} \bar{\sigma}^{\lambda}\right] & =2\left(g^{\mu \nu} g^{\rho \lambda}-g^{\mu \rho} g^{\nu \lambda}+g^{\nu \rho} g^{\mu \lambda}-i \varepsilon^{\mu \nu \rho \lambda}\right) .
\end{aligned}
$$

\section{A.2 Expansion of the Scalar Propagator Matrix}

We can now use the 4-dimensional basis of the generalized Pauli matrices $\sigma^{\mu}$ to expand the inverse propagator in (2.5) $)$, i.e.

$$
\Delta^{-1}(s)=\left(\begin{array}{cc}
s-M_{1}^{2} & -\operatorname{Im} \Pi_{12}^{\mathrm{abs}}(s) \\
\operatorname{Im} \Pi_{12}^{\mathrm{abs}}(s) & s-M_{2}^{2}
\end{array}\right)+i\left(\begin{array}{cc}
\Pi_{11}^{\mathrm{abs}}(s) & \operatorname{Re} \Pi_{12}^{\mathrm{abs}}(s) \\
\operatorname{Re} \Pi_{12}^{\mathrm{abs}}(s) & \Pi_{22}^{\mathrm{abs}}(s)
\end{array}\right) .
$$


To this end, we use (A.3) to write the $2 \times 2$ propagator matrix $\Delta(s)$ as

$$
\Delta(s)=\frac{\Delta_{\mu}^{-1}(s) \bar{\sigma}^{\mu}}{\operatorname{det}\left[\Delta^{-1}(s)\right]},
$$

where the complex 4 -vector components $\Delta_{\mu}^{-1}(s)$ are given by

$$
\begin{aligned}
\Delta_{0}^{-1}(s) & =s-\frac{1}{2}\left(M_{1}^{2}+M_{2}^{2}\right)+\frac{i}{2}\left[\Pi_{11}^{\mathrm{abs}}(s)+\Pi_{22}^{\mathrm{abs}}(s)\right], \\
\Delta_{1}^{-1}(s) & =i \operatorname{Re} \Pi_{12}^{\mathrm{abs}}(s) \\
\Delta_{2}^{-1}(s) & =-i \operatorname{Im} \Pi_{12}^{\mathrm{abs}}(s), \\
\Delta_{3}^{-1}(s) & =\frac{1}{2}\left(M_{2}^{2}-M_{1}^{2}\right)+\frac{i}{2}\left[\Pi_{11}^{\mathrm{abs}}(s)-\Pi_{22}^{\mathrm{abs}}(s)\right],
\end{aligned}
$$

and

$$
\begin{aligned}
\operatorname{det}\left[\Delta^{-1}(s)\right]= & \left(s-M_{1}^{2}\right)\left(s-M_{2}^{2}\right)+\left|\Pi_{12}^{\mathrm{abs}}(s)\right|^{2}-\Pi_{11}^{\mathrm{abs}}(s) \Pi_{22}^{\mathrm{abs}}(s) \\
& +i\left[\Pi_{11}^{\mathrm{abs}}(s)\left(s-M_{2}^{2}\right)+\Pi_{22}^{\mathrm{abs}}(s)\left(s-M_{1}^{2}\right)\right] .
\end{aligned}
$$

Correspondingly, the Hermitian decay matrices $T_{A, B, C}$ for the final states $A=\left(f_{k} \tilde{f}_{\tilde{l}}\right)$, see (2.12), etc., may be each decomposed as $T(s)=T_{\mu}(s) \sigma^{\mu}$, with the real coefficients

$$
\begin{aligned}
& T_{0}(s)=\frac{1}{2}\left[T^{11}(s)+T^{22}(s)\right], \\
& T_{1}(s)=\operatorname{Re} T^{12}(s), \\
& T_{2}(s)=-\operatorname{Im} T^{12}(s), \\
& T_{3}(s)=\frac{1}{2}\left[T^{11}(s)-T^{22}(s)\right] .
\end{aligned}
$$

where

$$
\begin{aligned}
& T^{12}(s)=\left(C_{k \tilde{l}}^{1, L *} C_{k \tilde{l}}^{2, L}+C_{k \tilde{l}}^{1, R *} C_{k \tilde{l}}^{2, R}\right)\left(s-m_{k}^{2}-m_{\tilde{l}}^{2}\right)-2\left(C_{k \tilde{l}}^{1, R *} C_{k \tilde{l}}^{2, L}+C_{k \tilde{l}}^{1, L *} C_{k \tilde{l}}^{2, R}\right) m_{k} m_{\tilde{l}}, \\
& T^{\alpha \alpha}(s)=\left(\left|C_{k \tilde{l}}^{\alpha, L *}\right|^{2}+\left|C_{k \tilde{l}}^{\alpha, R *}\right|^{2}\right)\left(s-m_{k}^{2}-m_{\tilde{l}}^{2}\right)-4 \operatorname{Re}\left(C_{k \tilde{l}}^{\alpha, L} C_{k \tilde{l}}^{\alpha, R *}\right) m_{k} m_{\tilde{l}} . \quad(\mathrm{A} .22)
\end{aligned}
$$

Note that the partial scalar decay widths at tree level are

$$
\Gamma_{\alpha}\left(\widetilde{S}_{\alpha} \rightarrow f_{k} \tilde{f}_{\tilde{l}}\right)=\frac{\lambda^{1 / 2}\left(M_{\alpha}^{2}, m_{k}^{2}, m_{\tilde{l}}^{2}\right)}{16 \pi M_{\alpha}^{3}} T^{\alpha \alpha}\left(M_{\alpha}^{2}\right)
$$




\section{References}

[1] A. Einstein, B. Podolsky and N. Rosen, Phys. Rev. 47 (1935) 777.

[2] J. S. Bell, "Speakable and Unspeakable in Quantum Mechanics," Cambridge University Press, 1987.

[3] J. Bernabeu, N. E. Mavromatos and J. Papavassiliou, Phys. Rev. Lett. 92 (2004) 131601 arXiv:hep-ph/0310180.

[4] A. Pilaftsis, Nucl. Phys. B 504, 61 (1997) arXiv:hep-ph/9702393.

[5] S. Y. Choi and M. Drees, Phys. Lett. B 435 (1998) 356 [arXiv:hep-ph/9805474];

S. Y. Choi, M. Drees, B. Gaissmaier and J. S. Lee, Phys. Rev. D 64 (2001) 095009 arXiv:hep-ph/0103284.

[6] H. E. Haber and G. L. Kane, Phys. Rept. 117, 75 (1985);

H. P. Nilles, Phys. Rept. 110 (1984) 1;

M. Drees, R. Godbole and P. Roy, Theory and phenomenology of sparticles, World Scientific, Singapore (2004).

[7] S. Heinemeyer, H. Rzehak and C. Schappacher, Phys. Rev. D 82 (2010) 075010 arXiv:1007.0689 [hep-ph]].

[8] H. Hlucha, H. Eberl and W. Frisch, arXiv:1104.2151 [hep-ph].

[9] A. Bartl, S. Hesselbach, K. Hidaka, T. Kernreiter and W. Porod, Phys. Rev. D 70, 035003 (2004) arXiv:hep-ph/0311338.

[10] H. Eberl, S. M. R. Frank and W. Majerotto, Eur. Phys. J. C 70, 1017 (2010) arXiv:0912.4675 [hep-ph]].

[11] R. E. Cutkosky, J. Math. Phys. 1, 429 (1960);

M. J. G. Veltman, "Diagrammatica: The Path to Feynman rules," Cambridge, UK: Univ. Pr. (1994) (Cambridge lecture notes in physics, 4).

[12] A. Pilaftsis and M. Nowakowski, Phys. Lett. B 245 (1990) 185.

[13] N. Arkani-Hamed, J. L. Feng, L. J. Hall and H. C. Cheng, Nucl. Phys. B 505 (1997) 3 arXiv:hep-ph/9704205];

D. Bowser-Chao and W. Y. Keung, Phys. Rev. D 56 (1997) 3924 arXiv:hep-ph/9704219.

[14] D. Binosi and J. Papavassiliou, Phys. Rept. 479 (2009) 1 [arXiv:0909.2536 [hep-ph]].

[15] F. F. Deppisch and O. Kittel, JHEP 1006, 067 (2010) arXiv:1003.5186 [hep-ph]]; JHEP 0909, 110 (2009) [Erratum-ibid. 1003, 091 (2010)] [arXiv:0905.3088 [hep-ph]].

[16] W. Beenakker, M. Kramer, T. Plehn, M. Spira and P. M. Zerwas, Nucl. Phys. B 515, 3 (1998) arXiv:hep-ph/9710451.

[17] O. Kittel, arXiv:hep-ph/0504183. 
[18] G. Moortgat-Pick, K. Rolbiecki and J. Tattersall, Phys. Rev. D 83, 115012 (2011) arXiv:1008.2206 [hep-ph]]; G. Moortgat-Pick, K. Rolbiecki, J. Tattersall and P. Wienemann, JHEP 1001, 004 (2010) [arXiv:0908.2631 [hep-ph]].

[19] J. R. Ellis, J. S. Lee and A. Pilaftsis, Phys. Rev. D 70 (2004) 075010 [arXiv:hep-ph/0404167]; Phys. Rev. D 72 (2005) 095006 [arXiv:hep-ph/0507046]; Phys. Rev. D 71 (2005) 075007 arXiv:hep-ph/0502251.

[20] J. Bernabeu, D. Binosi and J. Papavassiliou, JHEP 0609 (2006) 023 arXiv:hep-ph/0604046;

H. K. Dreiner, O. Kittel and F. von der Pahlen, JHEP 0801, 017 (2008) arXiv:0711.2253 [hep-ph]];

O. Kittel and F. von der Pahlen, JHEP 0808, 030 (2008) arXiv:0806.4534 [hep-ph]].

[21] S. Bray, J. S. Lee and A. Pilaftsis, Nucl. Phys. B 786 (2007) 95 arXiv:hep-ph/0702294];

S. Blanchet, Z. Chacko, S. S. Granor and R. N. Mohapatra, Phys. Rev. D 82 (2010) 076008 [arXiv:0904.2174 [hep-ph]]. 